\title{
Tratamiento de las malformaciones en extremidades en el síndrome de bridas amnióticas: a propósito de un caso
} Amniotic band syndrome: management of skeletal limb abnormalities. A case report

\author{
Dr. Enrique Guillén Botaya ${ }^{a}$ Dra. Laura Pino Almero ${ }^{a}$ Dra. María O. Molini Menchón ${ }^{b}$, \\ Dra. Virina González Alonsoc, Dr. Manuel Pérez-Montejano y Dra. M. Fe Minguez Rey ${ }^{a}$
}

\begin{abstract}
RESUMEN
El síndrome de bridas amnióticas es un complejo de anomalías congénitas causadas por la rotura prematura del amnios, por lo que se originan bandas fibrosas que comprimen determinadas regiones del feto. Afecta a entre 1:1200 y 1:15000 recién nacidos vivos según las series consultadas.

La clínica típica consiste en alteraciones en las extremidades (anillos de constricción, amputaciones asimétricas más o menos distales, sindactilias y pie zambo), y se han descrito alteraciones toracoabdominales o faciales. Recientes trabajos han demostrado la utilidad del método de Ponseti en el tratamiento del pie zambo asociado al síndrome de bridas amnióticas. Se presenta el caso de un recién nacido que padecía un síndrome de bridas amnióticas y se ahonda en el manejo y la resolución quirúrgica de las malformaciones en las extremidades. En especial, se analiza la utilización del método de Ponseti en el tratamiento del pie zambo congénito sindrómico.

Palabras clave: sindrome de bandas amnióticas, procedimientos quirúrgicos operativos, anomalías congénitas.
\end{abstract}

\begin{abstract}
Amniotic band syndrome consists in a group of congenital abnormalities caused by strands of the amniotic sac that entangle some parts of the fetus. Those strands result from premature rupture of amnios. The incidence of amniotic band syndrome is 1:1200 to 1:15,000 live births, depending on case studies. Mostly affected parts of fetus are limbs (asymmetric amputations, syndactyly and clubbed foot) but facial and thoracoabdominal abnormalities have also been described.

Recent works have proved the utility of Ponseti method to treat clubfoot associated with amniotic band syndrome. We report the case of a newborn with amniotic band syndrome focusing on management and surgical repair of limbs deformities. Especially, we highlight the use of Ponseti method in treatment of syndromic clubfoot.

Key words: amniotic band syndrome, operative surgical procedures, congenital abnormalities.
\end{abstract}

http: / / dx.doi.org/10.5546/ aap.2020.e486

a. Servicio de Cirugía Ortopédica y Traumatología.

b. Servicio de Pediatría.

c. Servicio de Cirugía Plástica y Reparadora.

Hospital Clínico Universitario-Malvarrosa, Valencia, España.

Correspondencia:

Dra. M. Fe Minguez Rey: mfeminguez@gmail.com

Financiamiento: Ninguno.

Conflicto de intereses: Ninguno que declarar.

Recibido: 27-1-2020

Aceptado: $31-3-2020$
Cómo citar: Guillén Botaya E, Pino Almero L, Molini Menchón MO, González Alonso V, et al. Tratamiento de las malformaciones en extremidades en el síndrome de bridas amnióticas: a propósito de un caso. Arch Argent Pediatr 2020;118(5):e486-e490.

\section{INTRODUCCIÓN}

El síndrome de bridas amnióticas (SBA) es un espectro de alteraciones congénitas caracterizadas por la existencia de bridas fibrosas corioamnióticas en la placenta que estrangulan regiones del feto, lo que provoca malformaciones en las extremidades o craneofaciales. Las alteraciones viscerales son poco frecuentes. ${ }^{1}$ Afecta a entre $1: 1200$ y $1: 15000$ nacidos vivos, ${ }^{2}$ y tiene carácter esporádico.

Las anomalías en las extremidades más frecuentes son los anillos de constricción con linfedema distal, las amputaciones, sindactilias y el pie zambo. El anillo de constricción es un surco de tipo cicatricial circunferencial y perpendicular al eje mayor del miembro afectado. En función de su gravedad, Patterson ${ }^{3}$ describió cuatro grados de afectación: el grado 1 consiste en un anillo simple con extremidad normal hacia distal; el grado 2 añade atrofia y linfedema distales, y, en el grado 3, el anillo se asocia a sindactilias en los dedos. Finalmente, se considera el grado 4 en los casos de amputación. A nivel digital, un mismo anillo puede rodear a más de un dedo y originar una pseudosindactilia, caracterizada por la fusión distal con la separación proximal de los dedos, lo que la diferencia de la verdadera sindactilia. ${ }^{4}$

A continuación, se relata un caso de SBA. Se ahonda en la resolución quirúrgica de las manifestaciones en las extremidades y se enfatiza en el tratamiento del pie zambo congénito sindrómico.

\section{CASO CLÍNICO}

Madre primípara caucásica de 20 años con embarazo controlado y padre caucásico sano, provenientes de familias con bajo nivel socioeconómico. En las ecografías del primer trimestre, se detectó, en el feto, una malformación 
en la extremidad inferior izquierda compatible con el síndrome por bridas. Los progenitores no referían exposiciones de riesgo, como metadona o ácido lisérgico. ${ }^{5}$

En la semana $34+1$, la madre ingresó en nuestro Centro por la rotura prematura de la membrana con estreptococo betahemolítico negativo. A las $34+6$ semanas, se produjo el nacimiento por parto vaginal eutócico del varón de 2190 gramos, $46 \mathrm{~cm}$ de longitud y $30 \mathrm{~cm}$ de perímetro cefálico. Se apreciaban deformidades en las cuatro extremidades: en ambas manos, existía una pseudosindactilia entre el tercer y el cuarto dedo (Figura 1), y, en el miembro inferior derecho, se apreciaba un anillo de constricción Patterson 1 en el tercio medio de la pantorrilla.

Además, existía un anillo profundo en el tercio distal del miembro inferior izquierdo con compromiso vascular asociado a un pie zambo, además de un marcado linfedema en el dorso del pie y acrosindactilia de los tres primeros dedos, por lo que se consideró Patterson 3 (Figura 2). Por lo demás, presentaba un fenotipo normal, sin otros rasgos sindrómicos: genitales externos normocoloreados de varón, ausencia de signos de disrafismo oculto.

Figura 1. Pseudosindactilia en el tercer y el cuarto dedo de la mano derecha al nacer

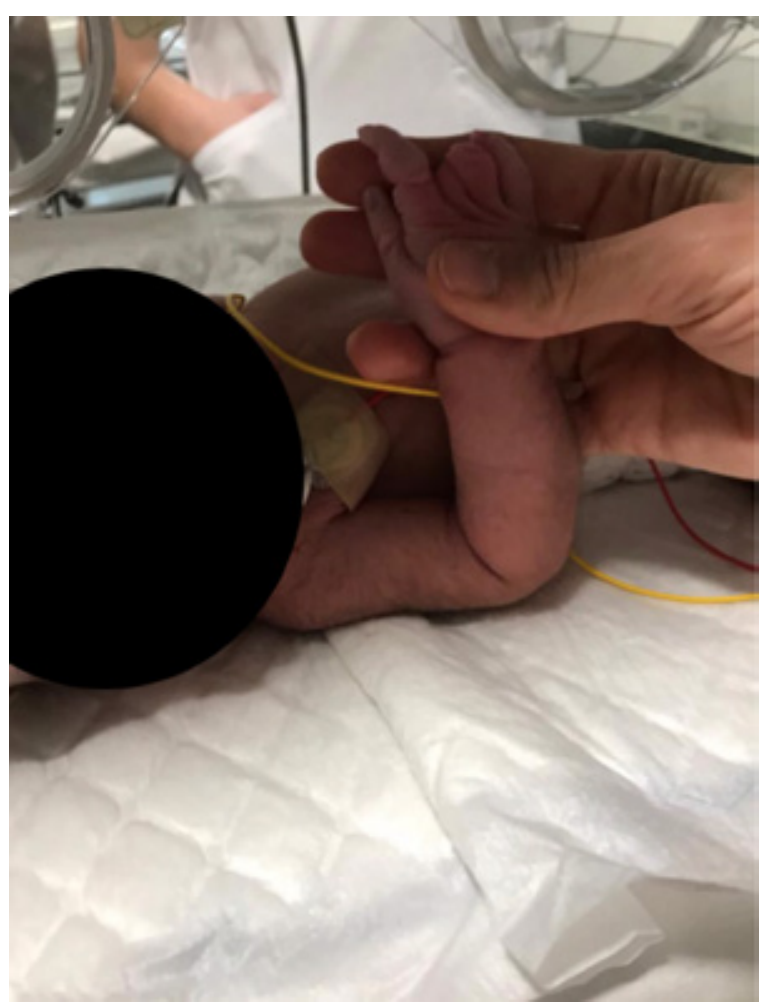

Se operó quirúrgicamente en la cuarta hora de vida para el salvamento del miembro inferior izquierdo, se extirpó la banda constrictora y se reconstruyeron los bordes con "W" plastias. El paciente fue dado de alta el décimo día.

Durante las revisiones sucesivas, la persistencia del linfedema en el dorso del pie izquierdo dificultaba el inicio del tratamiento precoz del pie zambo mediante el método de

Figura 2. Anillo de constricción en el miembro inferior izquierdo con compromiso vascular. Pie zambo con marcado linfedema en el dorso y acrosindactilia de los dedos

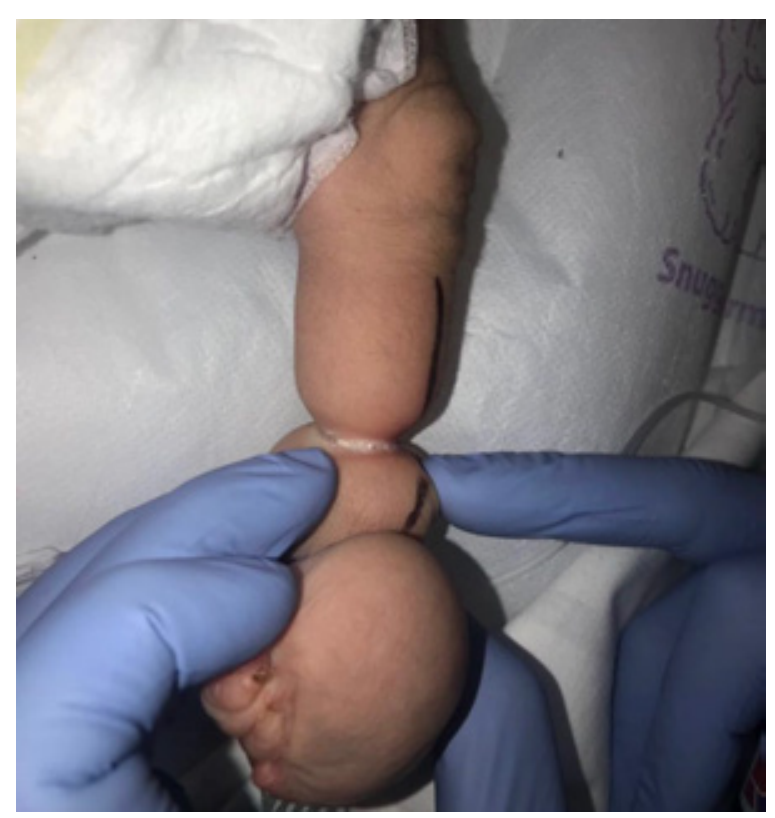

FIGURA 3. Imagen previa a la separación de la pseudosindactilia del tercer y cuarto dedo de la mano izquierda

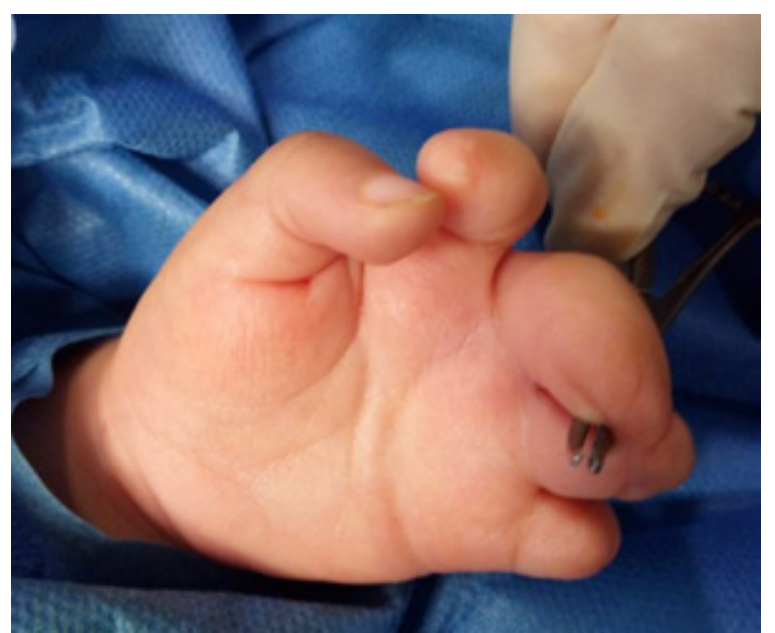


Ponseti. A los diez meses de vida, se extirpó la tumoración linfedematosa, además de resecar el anillo de constricción de la pierna derecha. También se separaron las pseudosindactilias entre el tercer y el cuarto dedo de ambas manos (Figura 3).

Tres semanas después de la intervención, se iniciaron las manipulaciones y yesos seriados para la corrección del pie zambo según el método de Ponseti (Figura 4. a). Tras seis yesos, se obtuvo una corrección completa, a excepción del equino, que requirió de la realización de una tenotomía percutánea del tendón de Aquiles. En la misma intervención, se separó la acrosindactilia del pie izquierdo y se inmovilizó el miembro con un yeso.

En la tercera semana posquirúrgica, se inició el tratamiento con la ortesis de DennisBrown durante 23 horas al día. La evolución fue satisfactoria, pese al cumplimiento irregular del tratamiento. Tras tres meses, se autorizó su retirada durante 12 horas al día. A los 24 meses de vida, el paciente presenta un pie plantígrado e indoloro (Figura 4. b), pero con tendencia a la supinación dinámica, por lo que puede precisar, en el futuro, una transposición del tibial anterior a la tercera cuña.

\section{DISCUSIÓN}

Según Patterson, ${ }^{3}$ el diagnóstico clínico del SBA debería incluir, al menos, dos de los siguientes criterios: anillo de constricción, con o sin deformidad distal y linfedema; fusiones distales o amputaciones digitales congénitas. Se ha de realizar el diagnóstico diferencial con el síndrome del cordón corto, que se debe a un defecto de cierre de la pared abdominal anterior fetal por bridas amnióticas, lo que provoca un cordón umbilical corto, gastrosquisis o anomalías vertebrales.

La presencia en nuestro paciente de anillos de constricción, con linfedema distal en el miembro inferior izquierdo y fusiones parciales de los dedos en las extremidades superiores e inferiores sin alteraciones del cordón umbilical, columna vertebral ni de la pared abdominal, hizo considerar como primera opción el SBA. Debido a la heterogeneidad de expresión de las malformaciones, es fundamental individualizar el tratamiento y considerar la funcionalidad sobre la base de la programación quirúrgica.

Con respecto a la exéresis de los anillos, la clasificación de Patterson tiene implicaciones en el tratamiento, pues se considera que los grados 2 y 3 precisan cirugía urgente para el salvamento

FIgURA 4. Tratamiento correctivo del pie zambo y evolución clínica del paciente

a) Corrección progresiva del pie zambo mediante yesos seriados según el método de Ponseti.

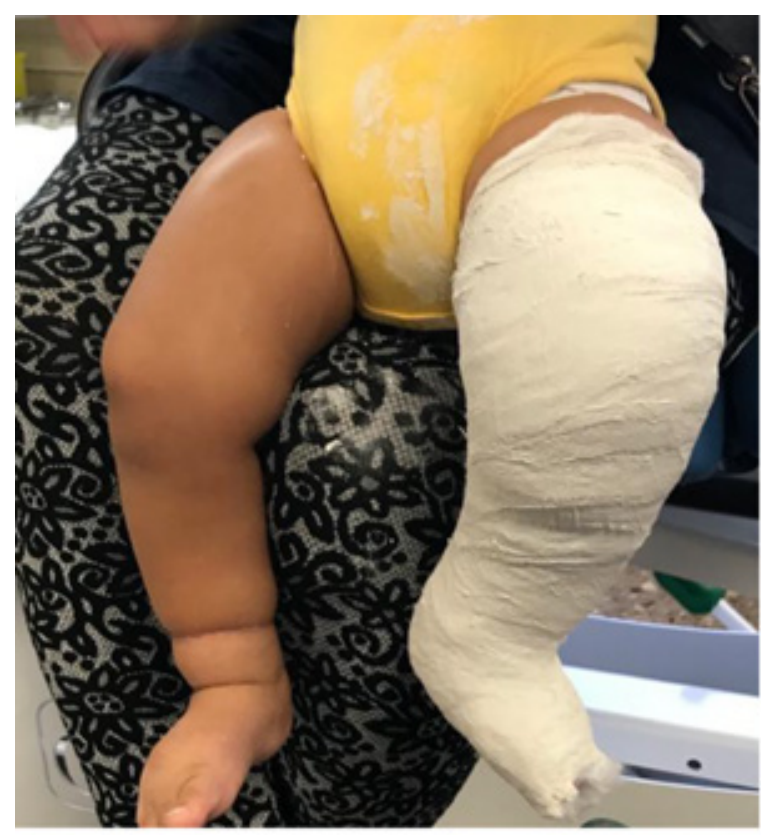

b) A los 24 meses de vida, pie izquierdo plantígrado, útil e indoloro, con ligera tendencia a la supinación dinámica.

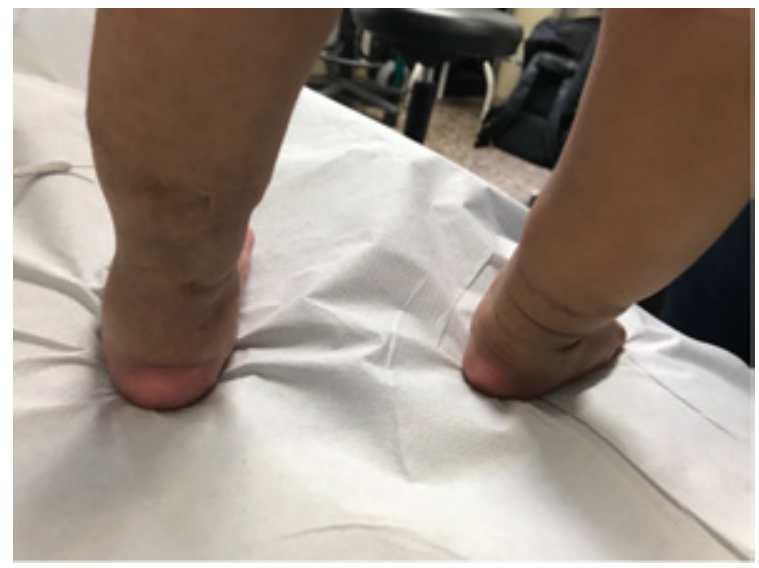


de la extremidad, y se puede diferir la cirugía en los casos de grado 1.6 Por este motivo, se intervino de forma urgente el miembro inferior izquierdo (Patterson 3), y se demoró la cirugía del anillo del miembro inferior derecho (Patterson 1).

En cuanto a la técnica de la liberación, la clásica consiste en escindirlos en una sola etapa mediante plastias circulares. ${ }^{7}$ En cambio, otros autores ${ }^{8}$ la realizan en dos fases para evitar el daño vascular: en una primera etapa, se descomprimen las estructuras vasculonerviosas y se completa la exéresis semanas después. En nuestro caso, se realizó una técnica en un solo paso en ambas piernas, dado que proporciona un excelente resultado, siempre y cuando se realice una disección cuidadosa para evitar el daño neurovascular. ${ }^{9}$

En cuanto a las sindactilias de la mano, se recomienda separarlas entre los 12 y los 18 meses, ${ }^{10}$ y se argumenta que la demora puede afectar la función de la mano, además de tener un gran impacto psicosocial para el paciente. En nuestro caso, se separaron a los 10 meses de vida, aprovechando el mismo tiempo quirúrgico de ambos miembros inferiores.

Históricamente, para el tratamiento del pie zambo asociado al SBA, se consideraba necesario realizar extensas cirugías abiertas para lograr pies plantígrados flexibles. ${ }^{11}$ No obstante, en la última década, se ha popularizado el método de Ponseti como tratamiento del pie zambo sindrómico, y se han obtenido buenos resultados. ${ }^{12}$

El método de Ponseti persigue corregir los componentes de la deformidad del pie zambo (equino, varo, aducto, supinado) mediante manipulaciones y yesos seriados, y reporta tasas de éxito de hasta el $97 \%$ para el pie zambo idiopático. ${ }^{13}$ Se recomienda iniciarlo en las dos primeras semanas de vida, aunque se han descrito pacientes tratados a los 10 años de vida, con peores resultados. ${ }^{14}$ Tras 4 o 5 manipulaciones, se logra la corrección total, y se precisa, ocasionalmente, de una tenotomía percutánea del tendón de Aquiles para corregir el equino residual. Posteriormente, se mantiene la corrección lograda con una ortesis en abducción (Dennis-Brown), que se retira de manera progresiva.

Zionts et al., ${ }^{15}$ publicaron una serie de 6 pacientes con pie zambo secundario a SBA tratados con el método de Ponseti. Precisaron de 6 yesos de media, y, en todos los casos, se realizó una tenotomía percutánea del tendón de Aquiles, que logró una corrección inicial del $100 \%$. Pese a que existió una recurrencia en 5 de ellos, asociada a la no utilización de la ortesis de Dennis-Brown, únicamente, precisó cirugía agresiva un paciente. Al finalizar el seguimiento, todos los pies eran flexibles y plantígrados.

Esta tendencia reciente se observa en nuestro caso, en el que se logró la corrección mediante 6 manipulaciones, y fue necesaria la tenotomía percutánea del tendón de Aquiles. Pese al irregular cumplimiento de la ortesis, a los 24 meses de seguimiento, el paciente tiene un apoyo plantígrado y flexible sin haber recurrido a una cirugía agresiva.

Es importante resaltar que la técnica de corrección del pie zambo sindrómico precisa, habitualmente, de un mayor número de manipulaciones y yesos que en el pie zambo idiopático, por la mayor rigidez de partes blandas y la demora del tratamiento. Este hecho se observa en nuestro caso, ya que el linfedema del pie demoró las manipulaciones, y se precisaron 6 yesos para corregir la deformidad, por los 4 o 5 descritos por Ponseti.

Es fundamental, por tanto, realizar un tratamiento secuencial individualizado en estos pacientes e identificar aquellas afecciones que precisan cirugía urgente, como son los anillos de constricción Patterson 2 y 3 . Además, se debe recalcar la utilidad del método de Ponseti para el tratamiento del pie zambo sindrómico, dado que evita extensas cirugías y presenta buenos resultados.

\section{REFERENCIAS}

1. Da RochaFP, Pires JA, Fagundes DJ, Da Cunha RS. Síndrome de bridas amnióticas. Relato de un caso de tratamiento quirúrgicoy revisión de la literatura. Cir Plast Iberolatinoam. 2013; 39(2):181-6.

2. Castejón Cruz ÓA, Castillo Pérez EA, Varela Carrasco GF, Oviedo Ayala EA, et al. Síndrome de bandas amnióticas asociado a Secuencia de Potter en el Hospital Regional del Sur, Choluteca, Honduras. Científica. 2016; 14(1):28-31.

3. Patterson TJ. Congenital ring constrictions. Br J Plast Surg. $1961 ; 14: 1-31$.

4. Flatt AE. Webbed fingers. Proc (Bayl Univ Med Cent). 2005; 18(1):26-37.

5. Werler MM, Louik C, Mitchell AA. Epidemiologic analysis of maternal factors and amniotic band defects. Birth Defects Res A Clin Mol Teratol. 2003; 67(1):68-72.

6. Hung NN. Congenital constriction ring in children: sine plasty combined with removal of fibrous groove and fasciotomy. J Child Orthop. 2012; 6(3):189-97.

7. Prasetyono TO, Sitorus AS. A review on the safety of onestage circumferential ring constriction release. Int Surg. 2015; 100(2):341-9.

8. Jaiman R, Gangopadhyay AN, Gupta DK, Srivastava P, et al. A child presented with bilateral congenital constriction ring in lower extremity: a case report. Cases J. 2009; 2:7772.

9. Komarcevic A, Pajic M, Djermanov M, Jovanovic M, et al. 
Single stage surgical treatment of amniotic band syndrome: case report. Srp Arh Celok Lek. 2016; 144(9-10):545-9.

10. Jordan D, Hindocha S, Dhital M, Saleh M, et al. The epidemiology, genetics and future management of syndactyly. Open Orthop J. 2012; 6:14-27.

11. Chang $\mathrm{CH}$, Huang SC. Clubfoot deformity in congenital constriction band syndrome: manifestations and treatment. J Formos Med Assoc. 1998; 97(5):328-34.

12. Agarwal A, Shaharyar A, Kumar A. Clubfoot associated with congenital constriction band: the Ponseti Method perspective. Foot Ankle Spec. 2015; 8(3):230-3.
13. Ganesan B, Luximon A, Al-Jumaily A, Balasankar SK, et al. Ponseti method in the management of clubfoot under 2 years of age: A systematic review. PLoS One. 2017; 12(6):e0178299.

14. Shah A, Mehta R, Aroojis A. The Ponseti method of clubfoot treatment in walking age children: is it effective? A study of 56 children from 1 to 10 years of age. J Pediatr Orthop B. 2019; 28(2):159-66.

15. Zionts LE, Habell B. The use of the ponseti method to treat clubfeet associated with congenital annular band syndrome. J Pediatr Orthop. 2013; 33(5):563-8. 